\title{
Pulmonary Mucormycosis (Zygomycosis) Presenting as an Infective Exacerbation of Chronic Obstructive Pulmonary Disease
}

\author{
Elena Koushiappi ${ }^{1}$, Ilias Porfyridis ${ }^{1}$, Christos Karagiannis $^{2}$, Tonia Adamide ${ }^{1}$, Andreas Georgiou ${ }^{1}$ \\ ${ }^{1}$ Department of Respiratory Medicine, Nicosia General Hospital, Cyprus \\ ${ }^{2}$ Department of Microbiology, Nicosia General Hospital, Cyprus
}

Received: 15/11/2018

Accepted: 24/11/2018

Published: $18 / 12 / 2018$

\begin{abstract}
How to cite this article: Koushiappi E, Porfyridis I, Karagiannis C, Adamide T, Georgiou A. Pulmonary mucormycosis (zygomycosis) presenting as an infective exacerbation of chronic obstructive pulmonary disease. EJCRIM 2018;5: doi:10.12890/2018_000995.
\end{abstract}

Conflicts of Interests: The Authors declare that there are no competing interests.

This article is licensed under a Commons Attribution Non-Commercial 4.0 License

\section{ABSTRACT}

Mucormycosis is a life-threatening fungal infection whose incidence has been rising recently, mainly due to the increasing use of immunosuppressive and corticosteroid treatment. In previous decades, mucormycosis was associated with a very poor prognosis as mortality was approximately $100 \%$. Mortality rates reported in recent literature have only slightly improved despite the availability of targeted therapy with amphotericin B. Pulmonary mucormycosis is characteristically encountered in severely immunocompromised hosts, while rhino-orbital disease is often seen in individuals with diabetes mellitus. We report a rare case of fulminant pulmonary mucormycosis as an exceptionally rare complication of corticosteroid treatment in a 76-year-old patient with chronic obstructive pulmonary disease (COPD) and diabetes. The patient had presented with typical symptoms of an infective COPD exacerbation. The interesting aspects of our case were the absence of malignancy or immunosuppression, the isolation of Rhizomucor species, and the fungal invasion of the pleura and pericardium. Unfortunately, our patient died on the 49th day of hospitalisation, despite appropriate treatment.

\section{LEARNING POINTS}

- Pulmonary mucormycosis in patients with known respiratory disease may mimic an exacerbation of their lung disease, thus delaying diagnosis.

- Pulmonary mucormycosis can complicate corticosteroid treatment in elderly individuals with other predisposing factors, which is an emerging clinical concern.

- Pulmonary mucormycosis remains a potentially fatal disease, although early diagnosis and appropriate medical and surgical management can improve outcomes.

\section{KEYWORDS}

Mucormycosis, zygomycosis, Rhizomucor, chronic obstructive pulmonary disease, diabetes mellitus

\section{CASE DESCRIPTION}

A 76-year-old Caucasian man with known chronic obstructive pulmonary disease (COPD) stage D was admitted with worsening dyspnoea, productive cough with yellowish-brown sputum and haemoptysis. The patient had recently received oral corticosteroids for a COPD exacerbation. His past medical history included hypertension, diabetes mellitus type 2 , atrial flutter and COPD requiring long-term oxygen therapy. He was a former smoker (100 pack years), did not consume any alcohol and had no known allergies. 
Clinical examination revealed tachycardia, use of accessory muscles, pursed lip breathing, a prolonged expiratory phase and reduced breath sounds on the left side. The patient was febrile with a Glasgow Coma Scale of 15/15, heart rate of 166 beats per minute and blood pressure of 120/85 mmHg. Initial investigations showed an elevated white blood cell (WBC) count of $25 \times 10^{9}$ (neutrophilia) and a C-reactive protein (CRP) of $143 \mathrm{mg} / \mathrm{l}$.

\section{Methods and Procedures}

The admission chest $x$-ray revealed consolidation in the left lower zone and blunting of the left costophrenic angle (Fig. 1). Intravenous piperacillin/tazobactam and ciprofloxacin were commenced due to recent hospital admissions (no known colonisations), followed by meropenem and vancomycin due to clinical deterioration. Further chest x-rays demonstrated cavity formation (Fig. 2). A chest CT scan revealed multiple cavities, especially in the left lower lobe, and a left-sided pleural effusion (Fig. 3). Bronchoscopy showed copious purulent secretions and bronchial mucosa inflammation, especially at LC2 and LB6.

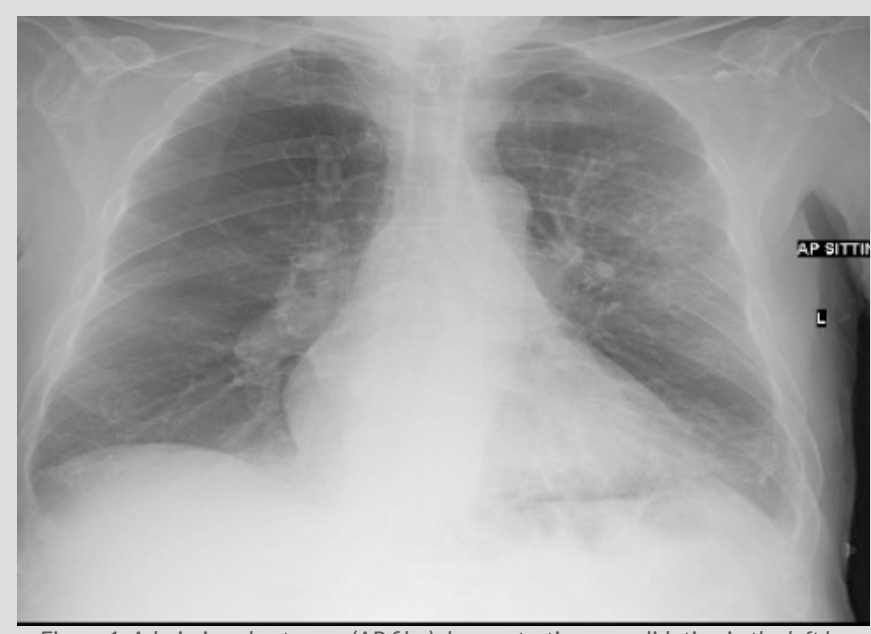

Figure 1. Admission chest $x$-ray (AP film) demonstrating consolidation in the left lower lobe and blunting of the left costophrenic angle

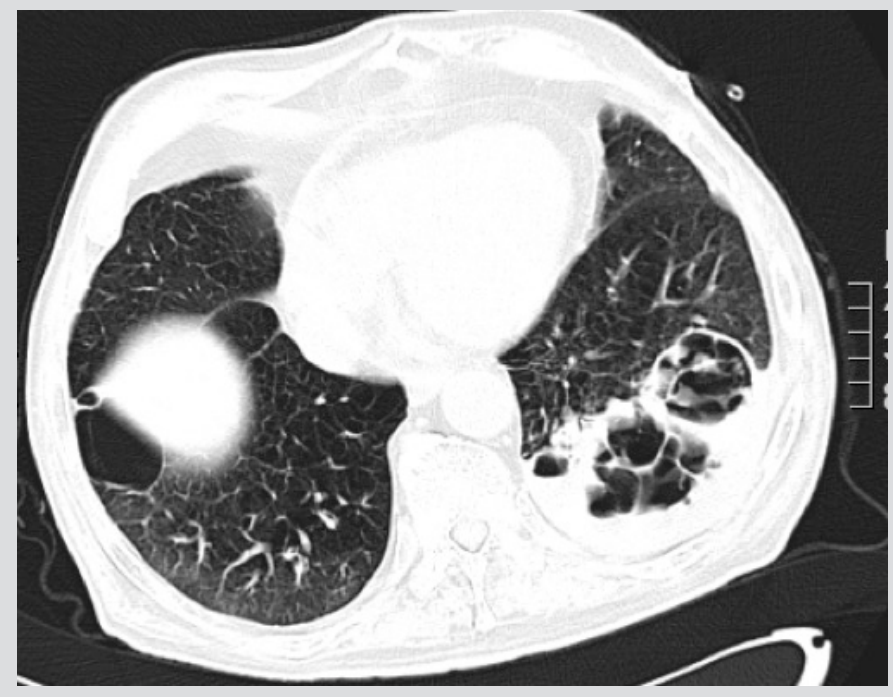

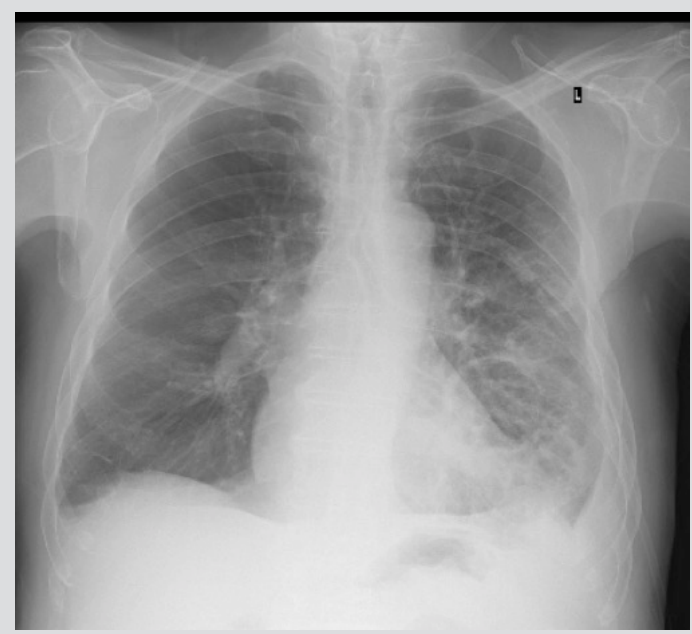

Figure 2. Subsequent chest $x$-ray (AP film) demonstrating multiple lung cavities, particularly in the left lower zone, and blunting of both costophrenic angles

Figure 3. A thorax CT scan demonstrating multiple cavity formation, particularly in the left lower lobe, and a left-sided pleural effusion

Sputum and bronchial washing cultures isolated a mould that grew rapidly on Sabouraud dextrose agar (SDA) $2 \%$ at $37^{\circ} \mathrm{C}$. The colony texture was cotton-candy like, velvety to floccose, with a white colour initially which, in time, turned yellowish-brown (Fig. 4). Microscopic examination in lactophenol cotton blue stain revealed long and hyaline hyphae, stolons that were not fully developed, and a few rhizoids of different sizes on stolons, between the sporangiophores (Fig. 5). 


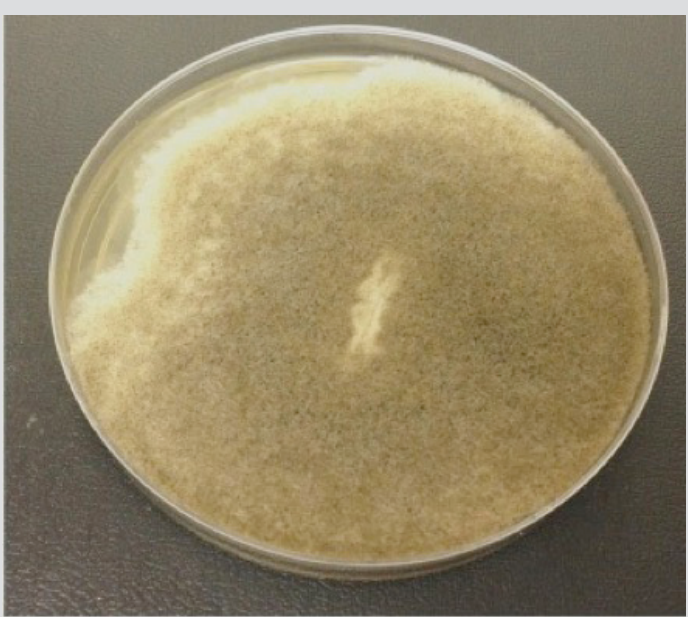

Figure 4. Mould growth on SDA 2\% showing a cotton, candy-like colony that was velvety to floccose and, in time, became yellowish-brown in colour

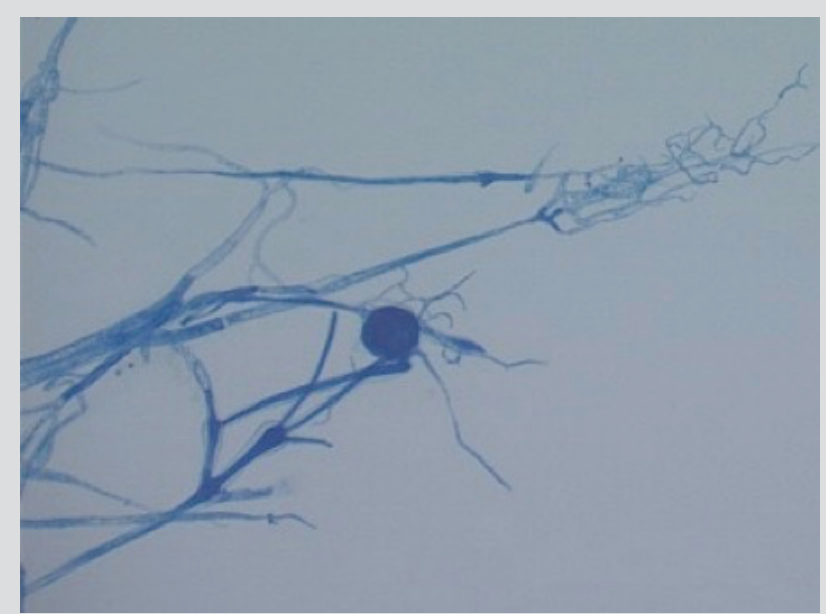

Figure 5. Lactophenol cotton blue stain demonstrating long and hyaline hyphae, stolons, rhizoids and sporangiophores

Sporangiophores were hyaline, simply branched, smooth-walled, variable and mostly subspherical. Sporangia were spherical with easily rupturing membranes and spherical columella. These findings were indicative of Rhizomucor species. The species grew on SDA $2 \%$ at $28^{\circ} \mathrm{C}$, $37^{\circ} \mathrm{C}$ and $42^{\circ} \mathrm{C}$. The isolate was also cultivated on potato dextrose agar (PDA) to obtain sporulation. For complete identification to subspecies level, the most appropriate method is polymerase chain reaction (PCR), but this is not currently available in our laboratory for moulds.

Blood and pleural fluid cultures were negative. Serum galactomannan antigen was negative. Bronchial washing cytology and bronchial biopsies were negative for malignancy. A rhino-orbital CT scan was normal. An echocardiogram demonstrated an ejection fraction of $50 \%$ and pericardial effusion $(1.3 \mathrm{~cm})$, with no valve disease.

Amphotericin B was commenced (on day 19). The patient's clinical course was complicated by pseudomembranous colitis, malnutrition and a hospital-acquired pneumonia (Klebsiella oxytoca and Pseudomonas aeruginosa isolated in subsequent sputum cultures). Despite appropriate antibiotic treatment, the patient died on the 49th day of hospitalization.

\section{DISCUSSION}

Mucormycosis (previously zygomycosis) is a rare fungal infection that has been increasing in frequency in recent years ${ }^{[1-5]}$. It is caused by fungi in the order of Mucorales, with most cases attributed to the genera of Rhizopus, Mucor, Lichtheimia and Rhizomucor ${ }^{[1-5]}$. These organisms are abundant in the environment, such as the soil and decaying matter. If inhaled, they can invade the tissues of susceptible patients. Having a strong affinity for vascular invasion, they can cause tissue necrosis and are thus associated with rapid disease progression and a high mortality rate ${ }^{[1]}$.

Immunodeficiency, as in haematological malignancies and stem cell or solid organ transplantations, is the principal predisposing factor ${ }^{[1-5]}$. Other conditions associated with mucormycosis include diabetes mellitus, trauma, burns, prematurity, corticosteroids, aplastic anaemia, iron overload, malnutrition, intravenous drug abuse, human immunodeficiency virus (HIV) and cytomegalovirus (CMV) infection ${ }^{[1-5]}$. There is no apparent age, sex or racial predilection. The typical sites of infection are rhino-orbito-cerebral, pulmonary, cutaneous and disseminated disease ${ }^{[1-5]}$.

The incidence is difficult to determine as it is not a notifiable disease and the risk differs amongst populations. Between 2005 and 2007 , a total of 230 possible cases were noted ${ }^{[5]}$. Of these, 28 were attributed to Rhizomucor species.

The principal host defence mechanism against the Mucorales fungi are the mononuclear and polymorphonuclear phagocytes, which produce cationic peptide defensins and generate oxidative metabolites ${ }^{[2]}$. High plasma glucose levels and acidosis, as in diabetes mellitus, reduce the ability of these phagocytes ${ }^{[2]}$. Acidosis is also associated with elevated serum iron levels, leading to rapid fungal proliferation ${ }^{[2]}$. Damage to endothelial cells leads to angioinvasion, vessel thrombosis, tissue necrosis and dissemination of the fungus. There is evidence that corticosteroid therapy causes functional defects in circulating neutrophils and impairs the role of bronchoalveolar macrophages in preventing spore germination following nasal inoculation ${ }^{[2]}$. 
CT scan findings in pulmonary mucormycosis (consolidation, nodules, cavities, pleural effusions, 'reverse halo sign') are not pathognomonic ${ }^{[1-3,6]}$. Microscopy reveals broad, non-septate hyphae, but these are fragile and can easily be destroyed if samples are not processed properly ${ }^{[1-3,6]}$. Cultures have a poor yield (30-50\%). PCR assays have recently provided promising results; however, they lack standardization and are therefore not widely employed ${ }^{[6]}$. Consequently, establishing the diagnosis can be challenging, and mucormycosis is often a post-mortem finding.

The mainstay of treatment is intravenous liposomal amphotericin $\mathrm{B}^{[1-5,7]}$. Surgical debridement or resection of affected tissues is recommended where feasible and is associated with better outcomes ${ }^{[5]}$. In our case, the patient was considered high risk for thoracic surgery.

Pulmonary mucormycosis carries a mortality rate of up to $87 \%$, while disseminated disease has a mortality rate of $90-100 \%[1,4,5,7,8]$. The poor outcomes may be partly due to inability to surgically resect the affected tissues. The high mortality rate may also reflect a delay in making the diagnosis and the severity of the predisposing conditions. Older patients have a worse prognosis, with a 1-year increase in age increasing mortality by $3 \%{ }^{[5]}$. Early treatment with amphotericin B and trauma as the underlying risk factor are associated with better survival rates ${ }^{[5]}$.

\section{CONCLUSION}

We describe a case of severe pulmonary mucormycosis with adjacent structure involvement (pleura, pericardium) in a nonimmunocompromised host. This case report describes the difficulty in establishing an early diagnosis, the relevant predisposing factors, the potential for rapid disease progression and disseminated disease, and the high mortality rate of this condition.

\section{REFERENCES}

1. Smith JA, Kauffman CA. Pulmonary fungal infections. Respirology 2012;17:913-926.

2. Spellberg B, Edwards J Jr, Ibrahim A. Novel perspectives on mucormycosis: pathophysiology, presentation and management. Clin Microbiol Rev 2005;18:556-569.

3. Gadkowske LB, Stout JE. Cavitary pulmonary disease. Clin Microbiol Rev 2008;21:305-333.

4. Roden MM, Zaoutis TE, Buchanan WL, Knudsen TA, Sarkisova TA, Schaufele RL, et al. Epidemiology and outcome of zygomycosis: a review of 929 reported cases. Clin Infect Dis 2005;42:634-653.

5. Skiada A, Pagano L, Groll A, Zimmerli S, Dupont B, Lagrou K, et al. Zygomycosis in Europe: analysis of 230 cases accrued by the registry of the European Confederation of Medical Mycology (ECMM) working group on zygomycosis between 2005 and 2007. Clin Microbiol Infect 2011:17:1859-1867.

6. Walsh TJ, Gamaletsou MN, McGinnis MR, Hayden RT, Kontoyiannis DP. Early clinical and laboratory diagnosis of invasive pulmonary, extrapulmonary and disseminated mucormycosis (zygomycosis). Clin Infect Dis 2012;54(S1):S55-S60.

7. Tedder M, Spratt JA, Anstadt MP, Hedge SS, Tedder SD, Lowe JE. Pulmonary mucormycosis: results of medical and surgical therapy. Ann Thorac Surg 1994;57:1044-1050.

8. Almyroudis NG, Sutton DA, Linden P, Rinaldi MG, Fung J, Kusne S. Zygomycosis in solid organ transplant recipients in a tertiary transplant centre and review of the literature. Am J Transplant 2006;6:2365-2374. 